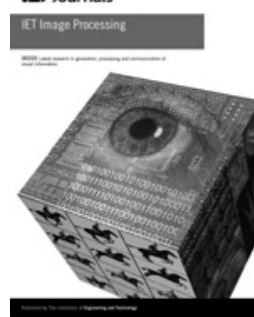

ISSN 1751-9659

\title{
Shape registration using characteristic functions
}

\author{
Muayed S. Al-Huseiny ${ }^{1,2}$, Sasan Mahmoodi ${ }^{1}$ \\ ${ }^{1}$ School of Electronic and Computer Science, University of Southampton, Southampton, UK \\ ${ }^{2}$ Electrical Engineering Department, College of Engineering, University of Wassit, Kut, Iraq \\ E-mail:sm3@ecs.soton.ac.uk
}

\begin{abstract}
This study presents a fast algorithm for the registration of shapes implicitly represented by their characteristic functions. The algorithm proposed here aims to recover the registration parameters (scaling, rotation and translation) by minimising a dissimilarity term between the two shapes. The proposed algorithm is based on phase correlation and statistical shape moments to compute the registration parameters individually. The registration method proposed here is applied to various registration problems, to address issues such as the registration of shapes with various topologies and registration of complex shapes containing various numbers of sub-shapes. The method proposed here is characterised with a better accuracy, a higher convergence speed, robustness at the presence of excessive noise and a better performance for registration over large databases of shapes, in comparison with other state-of-the-art shape registration techniques in the literature.
\end{abstract}

\section{Introduction}

Shape registration can be viewed as the result of a point-wise transformation between an observed source and a target shape. It is a fundamental task used to match two or more shapes taken, for example, at different times, from different viewpoints, or from different scenes. Most image analysis systems that evaluate images from various sources require the registration or a closely related operation as an intermediate step. Shape registration is an essential requirement shared among many computer vision domains and applications, such as pattern recognition, remote sensing, computer graphics and medical image analysis to name a few.

In shape registration, the representation of the shape plays a crucial role in the registration process, and can significantly influence the overall performance of the registration algorithm. Non-parametric shape representations such as the shape characteristic functions used in this paper are becoming a more popular choice, owing to their implicit handling of important shape deformation, and the simple extension to describe higher dimensions. Shape characteristic functions have previously been used in segmentation [1]. A dissimilarity measure based on shape characteristic functions is proposed in [2-4]. Such a dissimilarity measure has some favourable properties. First, it is independent of image size. This dissimilarity defines a distance which is non-negative, symmetric and satisfies the triangle inequality [5]. In this paper, the shape characteristic functions and their dissimilarity measure are exploited for shape registration.

Contour-based registration methods, examples of which are found in [6, 7], are among the techniques used widely in shape registration because of their fast convergence. These techniques, however, rely merely on the contour points representing the shapes to be matched during the registration. These techniques also require point correspondence for the boundary of the shapes. Consequently, contour-based methods fall short if the two shapes to be registered have different Euler characteristic numbers, or contain different number of sub-shapes, due to the ambiguity surrounding the process of establishing the point correspondence.

Signed distance function (SDF)-based shape registration techniques minimise the distance between the SDFs of two shapes [5, 8-11]. Some of these algorithms minimise such a distance by employing gradient descent algorithm (e.g. see $[5,8,9])$. These techniques are widely used with segmentation applications because it is relatively straightforward to embed such registration methods in functionals used for segmentation. The gradient descent-based shape registration methods (e.g. the seminal work in [8]) are in general capable of dealing with shapes with various Euler characteristic numbers (different topologies), although with the increase in shape complexity, the cost as well as the chances that these methods fall into local minima also increases. These methods, however, have some drawbacks: (i) low speed, (ii) in some cases, the instability and convergence to local minima, which leads to a limitation in the range of transformation these techniques can handle, and finally (iii) these methods are somewhat difficult to implement due to the need to tune the stopping parameters and time step for each transformation individually (see [5] for details). A shape registration method based on shape characteristic functions by using the iterated projection technique is also investigated in [12]. The drawback of such a method is that it is computationally expensive.

This paper presents a shape registration algorithm based on shape characteristic functions. The search space is assumed to 
be over shapes with similarity transformation function. Our algorithm therefore estimates the translation, rotation and scale parameters to minimise a dissimilarity measure. The algorithm proposed here employs orthogonal linear transformations and shape moments to estimate the registration parameters individually.

The 2D shape registration technique presented in [11] is another algorithm based on SDFs and is different from the work presented in this paper in the following ways:

(1) The registration technique proposed in [11] is based on SDFs and therefore the dissimilarity measure (e.g. see (2)) is theoretically unbounded for different (but similar) shapes with even optimal registration parameters. A pseudo-SDF is therefore proposed in [11] to resolve this issue in numerical implementations. However, the registration method proposed here does not suffer from such a theoretical issue, since a dissimilarity measure such as (2) is naturally bounded, if the shape characteristic functions are employed. (2) The registration algorithm proposed here is iterative and therefore its accuracy is always better than the accuracy of the non-iterative SDF-based registration scheme proposed in [11]. However, due to this iterative nature, the registration algorithm proposed here takes longer to converge to the optimal solution than the SDF-based algorithm considered in [11]. A numerical example is presented in Section 5.2 to show the superior performance of the registration algorithm proposed here in comparison to the work in [11].

(3) The desired rotational angle in [11] is estimated by minimising a term approximating the standard dissimilarity term (such as the one in (2)), whereas the exact dissimilarity term is minimised to estimate the desired rotational angle in the registration method proposed here.

A 3D shape registration approach is also proposed by Liu et al. [13] by using shape characteristic functions to eliminate theoretical issues associated with SDF functions encountered in the work presented in [11]. However, in contrast to the $2 \mathrm{D}$ registration method proposed here, before estimating the optimal parameters of the registration, the three axes of rotations need to be computed for $3 \mathrm{D}$ shape registration. Also contrary to the iterative 2D shape registration algorithm proposed here, the 3D shape registration algorithm of [13] is a non-iterative algorithm.

The main contributions of this paper are as follows:

(1) It employs the Parseval theorem to compute the translation and rotation parameters; however, we must stress that unlike early deployments of this theorem, such as in [14] and many others which are area-based methods, this paper presents a shape characteristic-based method where the characteristic functions of the shapes are used for registration. Our method also enjoys more flexibility in terms of handling a variety of source and target shapes in contrast with the registration method discussed in [14].

(2) The evaluation of the approach proposed here shows that this registration technique is robust, fast and suitable for a wide range of registration problems. These problems address issues such as shapes with various topologies (i.e. shapes with different Euler characteristic numbers) even at the presence of noise, and our method sustains a better performance over large databases. The results presented here are compared with the state-of-the-art shape registration algorithms in the literature.
The rest of the paper is structured as follows: The registration problem is stated in Section 2. The registration technique proposed here is presented in Section 3. The implementation issues are then discussed in Section 4. In this section, the implementation of the algorithm proposed in this paper is described in a form of a pseudo-code. The experimental results are presented in Section 5, and finally conclusions are drawn in Section 6.

\section{Statement of the problem}

Let $\phi_{p}(x, y): \Omega \rightarrow\{0,1\}$ and $\phi_{q}(x, y): \Omega \rightarrow\{0,1\}$ denote characteristic functions for shapes $p$ and $q$, where $\Omega$ is the bounded image domain. The characteristic functions are generally defined as

$$
\phi_{B}(x, y)= \begin{cases}1, & (x, y) \in I_{B} \\ 0, & (x, y) \in \Omega-\left(I_{B}+C\right)\end{cases}
$$

where $I_{B}$ is a subset of the domain $\Omega$ representing the interior of shape $B$ and $C$ is the shape boundary.

The parameters $s, \theta, T_{x}$ and $T_{y}$ representing scaling, rotation and translations in $x$ and $y$ directions, respectively, are required to transform $\phi_{p}$ to minimise a dissimilarity measure between $\phi_{p}$ and $\phi_{q}$ defined in (2)

$$
E=\iint_{\Omega}\left|\phi_{p}(x, y)-\phi_{q}\left(s R_{\theta}\left(x+T_{x}, y+T_{y}\right)\right)\right|^{2} \mathrm{~d} x \mathrm{~d} y
$$

so that

$$
\left(\hat{\theta}, \hat{s}, \hat{T}_{x}, \hat{T}_{y}\right)=\underset{\theta, s, T_{x}, T_{y}}{\arg \min } E
$$

where $\hat{\theta}, \hat{s}, \hat{T}_{x}$ and $\hat{T}_{y}$ are, respectively, the estimated angle, scale and translations. The functions $\phi_{p}, \phi_{q}$ and the transformed characteristic functions are in $\Omega$; that is, the aim is to compute registration parameters such that the result is still in $\Omega$. Also, $R_{\theta}$ is a conventional $2 \mathrm{D}$ rotation matrix

$$
R_{\theta}=\left[\begin{array}{cc}
\cos \theta & \sin \theta \\
-\sin \theta & \cos \theta
\end{array}\right]
$$

The minimisation of (2) in a gradient descent framework leads to a set of non-linear equations with respect to the desired parameters. In such a framework, the implementation of the equations obtained by vanishing the derivative of the dissimilarity measure with respect to registration parameters would be slow to converge, could fall into local minima and requires to continuously tune parameters for a smooth convergence. The objective of this paper is therefore to propose a method minimising distance terms equivalent to (2). The method should be robust against local minima, noise and also be fast, and easy to implement.

\section{Shape registration}

In this section, we discuss how to estimate registration parameters one by one by minimising the dissimilarity term (2). 


\subsection{Rotation}

The notion that a rotation in Cartesian domain is a displacement in the angular component of the polar coordinate system [15] is exploited here. To this end, the polar coordinate system is employed here in order to find the desirable rotation angle. Initially, the shape characteristic functions are centralised, that is

$$
\begin{aligned}
& \hat{\phi}_{p}(x, y)=\phi_{p}\left(x-p_{x}, y-p_{y}\right) \\
& \hat{\phi}_{q}(x, y)=\phi_{q}\left(x-q_{x}, y-q_{y}\right)
\end{aligned}
$$

where $\left(p_{x}, p_{y}\right)$ and $\left(q_{x}, q_{y}\right)$ are, respectively, the centroids of $\phi_{p}$ and $\phi_{q}$. The centralisation of shapes is necessary to find scaling and rotation parameters. This is due to the observation that for the rotation (or scaling) of a shape, the shape is initially transferred to its centroid. The rotation (or scaling) is then performed while the shape is in its centroid. The shape is finally transferred back from its centroid to its original location. We use this notion to calculate rotation and scaling parameters by centralising the shapes in (4) and (5).

A simple and efficient algorithm presented in [16] with sub-pixel accuracy is used to map $\hat{\phi}_{p}(x, y)$ and $\hat{\phi}_{q}(x, y)$ to polar coordinates. We then calculate $\hat{\phi}_{p}(\rho, \omega)$ and $\hat{\phi}_{q}(\rho, \omega)$ such that $x=\rho \cos \omega$ and $y=\rho \sin \omega$.

Let us initially define $\beta$ as a function of the desirable rotation angle $\theta$, that is

$$
\beta(\theta)=\iint_{\Omega}\left(\hat{\phi}_{q}(x, y) \hat{\phi}_{p}\left(R_{\theta}(x, y)\right)\right) \mathrm{d} x \mathrm{~d} y
$$

The desirable angle is estimated by minimising the dissimilarity term $E_{\theta}$ in (2) by assuming that the other transformation parameters are known and constant, that is

$$
E_{\theta}=\iint_{\Omega}\left|\hat{\phi}_{q}-\hat{\phi}_{p}\right|^{2} \mathrm{~d} x \mathrm{~d} y
$$

or

$$
\begin{aligned}
E_{\theta} & =\iint_{\Omega}\left(\left|\hat{\phi}_{q}-\hat{\phi}_{p}\right|\left|\hat{\phi}_{q}-\hat{\phi}_{p}\right|\right) \mathrm{d} x \mathrm{~d} y \\
& =\iint_{\Omega}\left|\hat{\phi}_{q}\right|^{2} \mathrm{~d} x \mathrm{~d} y-2\left\langle\hat{\phi}_{q} \hat{\phi}_{p}\right\rangle+\iint_{\Omega}\left|\hat{\phi}_{p}\right|^{2} \mathrm{~d} x \mathrm{~d} y
\end{aligned}
$$

The first and the last terms in the above equation are independent of $\theta$, therefore

$$
\begin{aligned}
\iint_{\Omega}\left|\hat{\phi}_{q}-\hat{\phi}_{p}\right|^{2} \mathrm{~d} x \mathrm{~d} y= & \iint_{\Omega}\left|\hat{\phi}_{q}\right|^{2} \mathrm{~d} x \mathrm{~d} y \\
& +\iint_{\Omega}\left|\hat{\phi}_{p}\right|^{2} \mathrm{~d} x \mathrm{~d} y-2 \beta
\end{aligned}
$$

Hence, the minimisation of (9) is achieved by maximising $\beta$. The optimal rotation angle is therefore calculated by maximising $\beta$ with respect to $\theta$, or

$$
\hat{\theta}=\underset{\theta}{\arg \max } \beta
$$

The maximum value for $\beta$ can be computed more easily when it is written in polar coordinates, that is

$$
\begin{array}{r}
\beta(\theta)=\iint_{\Omega}\left(\hat{\phi}_{q}(x, y) \hat{\phi}_{p}\left(R_{\theta}(x, y)\right)\right) \mathrm{d} x \mathrm{~d} y \\
=\iint_{\Omega}\left(\hat{\phi}_{q}(\rho, \omega) \hat{\phi}_{p}(\rho, \omega-\theta)\right) \rho \mathrm{d} \rho \mathrm{d} \omega
\end{array}
$$

By using the Parseval theorem and Fourier transform, we estimate the optimal value for $\theta$ by maximising $\beta$.

Let the Fourier transform of $\hat{\phi}_{q}$ and $\hat{\phi}_{p}$ be $\hat{\psi}_{q}$ and $\hat{\psi}_{p}$, respectively, such that

$$
\begin{aligned}
& \hat{\psi}_{p}(\eta, \xi)=\frac{1}{2 \pi} \iint \hat{\phi}_{p}(\rho, \omega) \mathrm{e}^{-i(\eta \rho+\omega \xi)} \mathrm{d} \rho \mathrm{d} \omega \\
& \hat{\psi}_{q}(\eta, \xi)=\frac{1}{2 \pi} \iint \hat{\phi}_{q}(\rho, \omega) \mathrm{e}^{-i(\eta \rho+\omega \xi)} \mathrm{d} \rho \mathrm{d} \omega
\end{aligned}
$$

where $\eta$ and $\xi$ are spatial frequencies in the polar coordinate system. Accordingly, by using Parseval's theorem, one can write

$$
\begin{array}{r}
\beta(\theta)=\iint_{\Omega}\left(\hat{\phi}_{q}(\rho, \omega) \hat{\phi}_{p}(\rho, \omega-\theta)\right) \rho \mathrm{d} \rho \mathrm{d} \omega \\
=i \iint\left(\frac{\partial \hat{\psi}_{q}(\eta, \xi)}{\partial \eta} \hat{\psi}_{p}^{*}(\eta, \xi) \mathrm{e}^{i(\xi \theta)}\right) \mathrm{d} \eta \mathrm{d} \xi
\end{array}
$$

where $(*)$ denotes the complex conjugate. Hence, $\hat{\theta}$ is computed as

$$
\begin{aligned}
\hat{\theta} & =\underset{\theta}{\arg \max } \beta \\
& =\underset{\theta}{\arg \max i \iint}\left(\frac{\partial \hat{\psi}_{q}(\eta, \xi)}{\partial \eta} \hat{\psi}_{p}^{*}(\eta, \xi) \mathrm{e}^{i(\xi \theta)}\right) \mathrm{d} \eta \mathrm{d} \xi
\end{aligned}
$$

Therefore, in the algorithm proposed here, the rotation angle is computed by using (14).

\subsection{Scale}

Similar to the previous section, it is possible to express shapes $p$ and $q$ by using some orthogonal shape moments such as Chebyshev moments and then rewrite distance term (2) with respect to these shape moments by using the Parseval theorem. However, a simpler approach, which is less numerically expensive and easier to handle, is proposed in this section to estimate the scaling parameter $s$. This approach calculates the scaling parameter between two shapes, by minimising the distance between their radial moments.

The radial moments of the source characteristic function $\hat{\phi}_{p}$ and the target characteristic function $\hat{\phi}_{q}$ are computed in (15) and (16)

$$
\begin{aligned}
& M_{m}^{q}=\iint_{\Omega}\left(\sqrt{x^{2}+y^{2}}\right)^{m} \hat{\phi}_{q}(x, y) \mathrm{d} x \mathrm{~d} y \\
& M_{m}^{p}=\iint_{\Omega}\left(\sqrt{x^{2}+y^{2}}\right)^{m} \hat{\phi}_{p}(x, y) \mathrm{d} x \mathrm{~d} y
\end{aligned}
$$

where $m$ represents the degree of the moment. The moments 
of a shape scaled by $1 / s$ are then calculated as

$$
M_{m}^{p / s}=\iint_{\Omega}\left(\sqrt{x^{2}+y^{2}}\right)^{m} \hat{\phi}_{p}\left(\frac{x}{s}, \frac{y}{s}\right) \mathrm{d} x \mathrm{~d} y
$$

By changing variables, that is, $X=x / s$, and $Y=y / s,(17)$ can be written as

$$
\begin{aligned}
M_{m}^{p / s} & =\iint_{\Omega} s^{m}\left(\sqrt{X^{2}+Y^{2}}\right)^{m} \hat{\phi}_{p}(X, Y) s^{2} \mathrm{~d} X \mathrm{~d} Y \\
& =s^{(m+2)} \iint_{\Omega}\left(\sqrt{X^{2}+Y^{2}}\right)^{m} \hat{\phi}_{p}(X, Y) \mathrm{d} X \mathrm{~d} Y \\
& =s^{(m+2)} M_{m}^{p}
\end{aligned}
$$

In order to minimise the distance between the two characteristic functions, we minimise the distance between their radial moments, that is

$$
E_{s}=\sum_{m}\left|M_{m}^{q}-M_{m}^{p / s}\right|^{2}=\sum_{m}\left|M_{m}^{q}-s^{m+2} M_{m}^{p}\right|^{2}
$$

The optimal scaling parameter is a minimiser of (18). To find the optimal scale, the derivative of $E_{s}$ with respect to $s$ should vanish, that is

$$
\sum_{m}\left[(m+2) M_{m}^{p} M_{m}^{q} s^{m+1}-(m+2)\left(M_{m}^{p}\right)^{2} s^{2 m+3}\right]=0
$$

The optimal scale is calculated by numerically solving the above polynomial equation.

\subsection{Translation}

By using the scaling and rotation parameters calculated in Sections 3.1 and 3.2 , the term (2) is optimised to calculate the translation parameters $T_{x}$ and $T_{y}$.

By employing the same approach explained in Section 3.1, the translation parameters are also estimated. The only difference here is that since the translations are shifts in the Cartesian coordinate systems, there is no need to represent the shape characteristic functions in the polar coordinate system.

Also, let us define $\delta$ as a function of the translation parameters $T_{x}$ and $T_{y}$, i.e.

$$
\delta\left(T_{x}, T_{y}\right)=\int\left(\phi_{q}(x, y) \phi_{p}\left(x-T_{x}, y-T_{y}\right)\right) \mathrm{d} x \mathrm{~d} y
$$

The unknown desired translation parameters will be estimated by minimising the dissimilarity term in (21)

$$
\begin{aligned}
\int_{x, y}\left|\phi_{q}-\phi_{p}\right|^{2} \mathrm{~d} x \mathrm{~d} y= & \int_{x, y}\left(\left|\phi_{q}-\phi_{p}\right|\left|\phi_{q}-\phi_{p}\right|\right) \mathrm{d} x \mathrm{~d} y \\
= & \int_{x, y} \phi_{q} \phi_{q} \mathrm{~d} x \mathrm{~d} y-\int_{x, y} \phi_{q} \phi_{p} \mathrm{~d} x \mathrm{~d} y \\
& -\int_{x, y} \phi_{q} \phi_{p} \mathrm{~d} x \mathrm{~d} y+\int_{x, y} \phi_{p} \phi_{p} \mathrm{~d} x \mathrm{~d} y \\
= & \int_{x, y}\left|\phi_{q}\right|^{2} \mathrm{~d} x \mathrm{~d} y-2\left\langle\phi_{q} \phi_{p}\right\rangle \\
& +\int_{x, y}\left|\phi_{p}\right|^{2} \mathrm{~d} x \mathrm{~d} y
\end{aligned}
$$

The first and the last terms in the above equation are independent of the translation parameters

The minimisation of (21) is therefore achieved by maximising $\left\langle\phi_{q} \phi_{p}\right\rangle$, that is

$$
\hat{T}_{x}, \hat{T}_{y}=\underset{T_{x}, T_{y}}{\arg \max }\left\langle\phi_{q} \phi_{p}\right\rangle=\underset{T_{x}, T_{y}}{\arg \max } \delta\left(T_{x}, T_{y}\right)
$$

Similar to what is presented in Section 3.1, the maximum value for $\left\langle\phi_{q} \phi_{p}\right\rangle$ is computed in the Fourier domain by using the Parseval theorem, that is

$$
\begin{aligned}
\left\lfloor\begin{array}{ll}
\hat{T}_{x} & \hat{T}_{y}
\end{array}\right\rfloor & =\underset{T_{x}, T_{y}}{\arg \max }\left\langle\phi_{q} \phi_{p}\right\rangle=\underset{T_{x}, T_{y}}{\arg \max } \int_{\omega_{x}} \int_{\omega_{y}} \\
& \left(\psi_{q}\left(\omega_{x}, \omega_{y}\right) \psi_{p}^{*}\left(\omega_{x}, \omega_{y}\right) \mathrm{e}^{i\left(T_{x} \omega_{x}+T_{y} \omega_{y}\right)}\right) \mathrm{d} \omega_{x} \mathrm{~d} \omega_{y}
\end{aligned}
$$

where $\hat{T}_{x}, \hat{T}_{y} \psi_{q}\left(\omega_{x}, \omega_{y}\right), \psi_{p}\left(\omega_{x}, \omega_{y}\right), \omega_{x}$ and $\omega_{y}$ represent the estimated optimal translation parameters, the 2D Fourier transform of $\phi_{q}$ and $\phi_{p}$, and the spatial frequencies, respectively. Equation (23) is thus used to compute the optimal translation parameters in the algorithm proposed here.

\section{Implementation issues}

An iterative scheme is used for the implementation of the algorithm proposed here. The registration parameters estimated by using (14), (19) and (23) are updated iteratively, until the difference between two consecutive registered shapes becomes less than a certain threshold. Therefore, (14), (19) and (23) can be used to form an iterative algorithm as follows:

At iteration $t$ :

- Centralise shapes $\left(\phi_{p}\right)_{t}$ and $\phi_{q}$

- Update the transformation parameters by optimising the following terms:

$$
\begin{gathered}
\hat{\theta}_{t+1}=\underset{\theta}{\arg \max } \beta=\underset{\theta}{\arg \max i} \\
\iint\left(\frac{\partial \hat{\psi}_{q}(\eta, \xi)}{\partial \eta}\left(\hat{\psi}_{p}^{*}(\eta, \xi)\right)_{t} \mathrm{e}^{i(\xi \theta)}\right) \mathrm{d} \eta \mathrm{d} \xi \\
\hat{s}_{t+1}=\underset{s}{\arg \max } \sum_{m}\left|M_{m}^{q}-s^{m+2}\left(M_{m}^{p}\right)_{t}\right|^{2} \\
\left\lfloor\left(\hat{T}_{x}\right)_{t+1}\left(\hat{T}_{y}\right)_{t+1}\right\rfloor=\underset{T_{x}, T_{y}}{\arg \max } \int_{\omega_{x}} \int_{\omega_{y}} \\
\left(\psi_{q}\left(\omega_{x}, \omega_{y}\right)\left(\psi_{p}^{*}\left(\omega_{x}, \omega_{y}\right)\right)_{t} \mathrm{e}^{i\left(T_{x} \omega_{x}+T_{y} \omega_{y}\right)}\right) \mathrm{d} \omega_{x} \mathrm{~d} \omega_{y}
\end{gathered}
$$

- Repeat the above steps, until the distance between $\left(\phi_{p}\right)_{t+1}$ and $\left(\phi_{p}\right)_{t}$ is less than a threshold.

In practice, two iterations are required for our registration algorithm to converge in the most of the experiments performed in this paper.

In our implementation, we use 'roots' built-in function in MATLAB to find the solutions of (19). We use up to the fifth radial moments $(m=5)$. Therefore in various examples 
on which our algorithm is applied, (19) has 13 roots. Ten roots are always complex, one is zero and one is negative. There is only one positive root which is the desired scale we use for registration.

For the implementation of (14), we take the following steps: At iteration $t$ :

(i) Calculate 2D Fourier transforms $\left(\hat{\psi}_{p}(\eta, \xi)\right)_{t}$ and $\hat{\psi}_{q}(\eta, \xi)$ of two shapes $\left(\phi_{p}\right)_{t}$ and $\phi_{q}$ in the polar coordinate system.

(ii) Calculate $\frac{\partial \hat{\psi}_{q}(\eta, \xi)}{\partial \eta}$.

(iii) Calculate the product of $\frac{\partial \hat{\psi}_{q}(\eta, \xi)}{\partial \eta}$ and $\left(\hat{\psi}_{p}^{*}(\eta, \xi)\right)_{t}$ pixel
by pixel. (iv) Take the inverse Fourier transform of the product calculated in (iii).

(v) Find the value (for $\theta$ ) in which the inverse Fourier transform calculated in (iv) is maximum for $\rho=0$.

Equation (23) is also implemented as explained in the following steps:

At iteration $t$ :

(i) Calculate $2 \mathrm{D}$ Fourier transforms $\left(\psi_{p}\left(\omega_{x}, \omega_{y}\right)\right)_{t}$ and $\psi_{q}\left(\omega_{x}\right.$, $\left.\omega_{y}\right)$ of two shapes $\left(\phi_{p}\right)_{t}$ and $\phi_{q}$ in the Cartesian coordinate system.

(ii) Calculate the product of $\left(\psi_{p}^{*}\left(\omega_{x}, \omega_{y}\right)\right)_{t}$ and $\psi_{q}\left(\omega_{x}, \omega_{y}\right)$ pixel by pixel.

(iii) Take the inverse Fourier transform of the product calculated in (ii).

(iv) Find the values (for $T_{x}$ and $T_{y}$ ) which maximises the function calculated in (iii).

It is noted that all registration algorithms discussed in this paper are implemented in a MATLAB (version 7) environment on a PC with a dual Intel processor each with $2.67 \mathrm{GHz}$ frequency.

\section{$5 \quad$ Results and discussions}

In this section, a set of examples is presented to demonstrate the performance of the shape registration method proposed here in comparison with other known registration methods in various shape registration problems. In all of the experiments presented in this paper, the moments up to the fifth degree are used to compute the scale parameter $s$ by using the proposed method in Section 3.2. The higher the degree of the employed moments is, the more accurately $s$ is estimated. However, this higher accuracy comes at the expense of higher numerical complexity. The results of the technique proposed here are compared with four well-known shape registration methods in this paper. The first method is based on contours representing shapes (e.g. see [7]). This method is referred to as the contour-based method throughout this section. Another method used here for comparison employs SDFs to represent shapes. A gradient descent approach is employed to compute the optimal solutions (see [8] for more details). Throughout this section the method is termed as the SDF-based method. The third method compared with our results here is the registration method based on the iterated projection method presented in [12]. Finally, we compare the performance of our algorithm with that of a non-iterative registration algorithm based on SDFs presented in [11].

In most of the examples, a distance term Dist is used to measure the quality of registration. This term is defined as: $\operatorname{Dist}\left(\phi_{\text {source }}, \phi_{\text {target }}\right)=\iint_{x} \mid \phi_{y}$ source $-\left.\phi_{\text {target }}\right|^{2} \mathrm{~d} x \mathrm{~d} y \quad$ where $\phi_{\text {source }}$ and $\phi_{\text {target }}$ are the shape characteristic functions of the two shapes to be registered. In other words, this distance counts the number of un-overlapped pixels in both shapes. Also the running time $(t)$ required to compute the optimal registration parameters is used as a measure of quality. For better visual demonstration, in the most of examples in this paper, the contours of the source and target shapes rather than their characteristic functions are shown in the figures throughout this section.

\subsection{Shapes with different topologies}

In the example presented in this sub-section, two shapes with different Euler characteristic numbers are used. These shapes obviously have different topologies. In Fig. 1a, the observed shape is an open ' 4 ' with Euler number one, and the target shape is a closed '4' with Euler number zero.

As shown in Fig. $1 b$, the two shapes are completely misaligned by using the contour-based method in [7]. This shortcoming stems directly from the necessity to establish correct point correspondence in order for this method to work correctly. Such a requirement makes the algorithm sensitive to topological changes due to the ambiguity of establishing point correspondences in certain cases. Since in this case the shapes have different topologies, such correspondence may not be achievable. Fig. 1c depicts the result of applying the registration method in [8] to solve the registration problem. This figure shows a typical example of the local minima hurdle associated with this approach. In Fig. $1 d$, the shapes in question are correctly registered by using the approach proposed in this paper. This better result can be attributed to the fact that the proposed approach exploits the entire region bounded by the perimeter to maximise the overlap between the two shapes. The
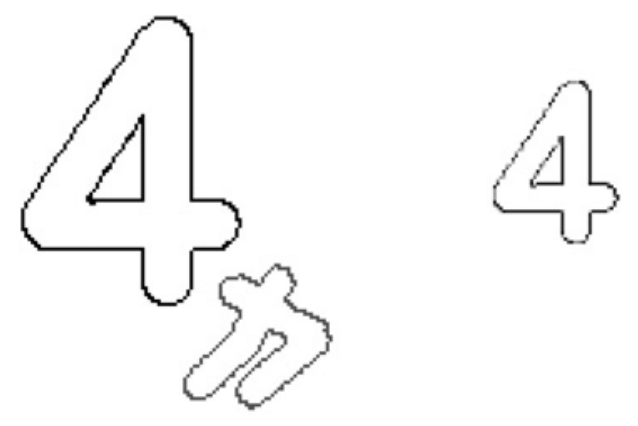

$a$

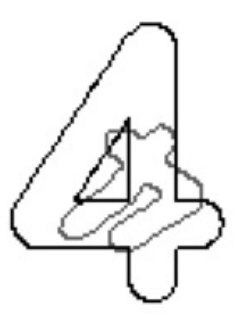

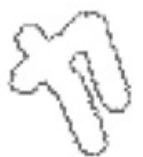

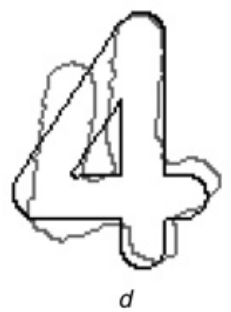

Fig. 1 Registration of shapes with different topologies

$a$ Initial shapes

$b$ the result of attempting to register the shapes using the contour based method in [7]

$c$ the registration of the shapes by using the SDF-based method in [8]

$d$ the two shapes superimposed optimally using the approach proposed here 
quantitative analysis associated with this experiment is listed in Table 1.

\subsection{Complex shapes containing various sub-shapes}

In the second example shown in Fig. 2, two complex shapes, each having different number of components are registered. The employment of contour method in [7] to register such shapes for example by registering the individual sub-shapes in the source shape to their counterparts in the target shape may lead to a partial registration. The sub-shapes with no counterparts therefore remain unregistered and the overall shape integrity may be distorted. The algorithm in [8], on the other hand, is capable of dealing with complex shapes.

However, by increasing the shape complexity, the chance of falling into local minima increases as well. The time required to find the final parameters as demonstrated in Table 1 becomes also longer. In Fig. 2, the observed shape is a clock face with conventional indicators, while the target shape has compass point indicators.

Similarly, in Fig. 3, a complex shape containing the bone shapes (sub-shapes) of an un-matured hand is registered to another complex shape containing the bone shapes (sub-shapes) of a matured one. We notice that the number of sub-shapes (bones) in these two complex shapes is different.

Table 1 Numerical comparisons (similarity distance and time spent by the processor to register two shapes) among some registration algorithms in the literature and the method proposed in this paper

\begin{tabular}{lccccccc}
\hline & Dist-Original. & $\begin{array}{c}\text { Dist-in [7] } \\
\text { (Pixel) }\end{array}$ & $\begin{array}{c}\text { Dist- in [8] } \\
\text { (Pixel) }\end{array}$ & $\begin{array}{c}\text { Dist-Proposed } \\
\text { (Pixel) }\end{array}$ & $\begin{array}{c}\text { Time-in [7] } \\
\text { (Sec.) }\end{array}$ & $\begin{array}{c}\text { Time-in [8] } \\
\text { (Sec.) }\end{array}$ & $\begin{array}{c}\text { Time-Proposed } \\
\text { (Sec.) }\end{array}$ \\
\hline number 4 & 3664 & 2296 & 2722 & 1078 & 1.09 & 28.25 & 2.83 \\
clock & 5859 & 5162 & 6442 & 4775 & 3.13 & 29.18 & 9.361 \\
hands-radiography & 8140 & 8303 & 8191 & 6194 & 3.15 & 1175.8 & 4.86 \\
similar-hands-1 & 7249 & 668 & 916 & 171 & 1.34 & 3543.5 & 2.89 \\
similar hands-2 & 13935 & 894 & 814 & 200 & 1.57 & 4712.5 & 2.81 \\
vertebrae-x-ray & Av. $=2244$ & Av. $=345.2$ & Av. $=862.4$ & Av. $=339.2$ & Av. $=1.05$ & Av. $=470.5$ & Av. $=3.06$ \\
\hline
\end{tabular}

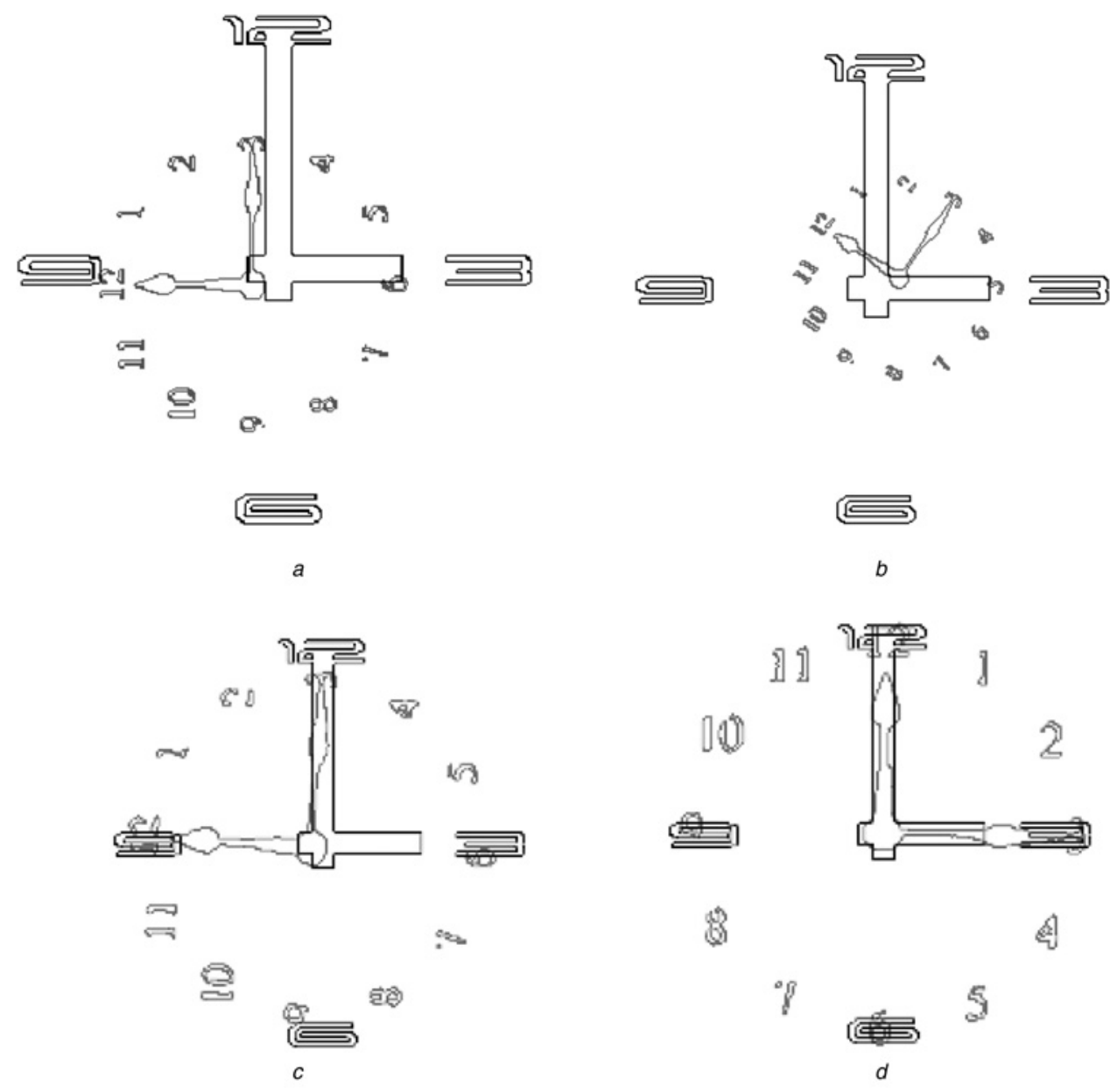

Fig. 2 Registration of shapes with different number of components

$a$ Initial shapes

$b$ Registration using contour-based technique in [7]

$c$ Registration using the SDF-based algorithm proposed in [8]

$d$ Registration using the algorithm proposed here 

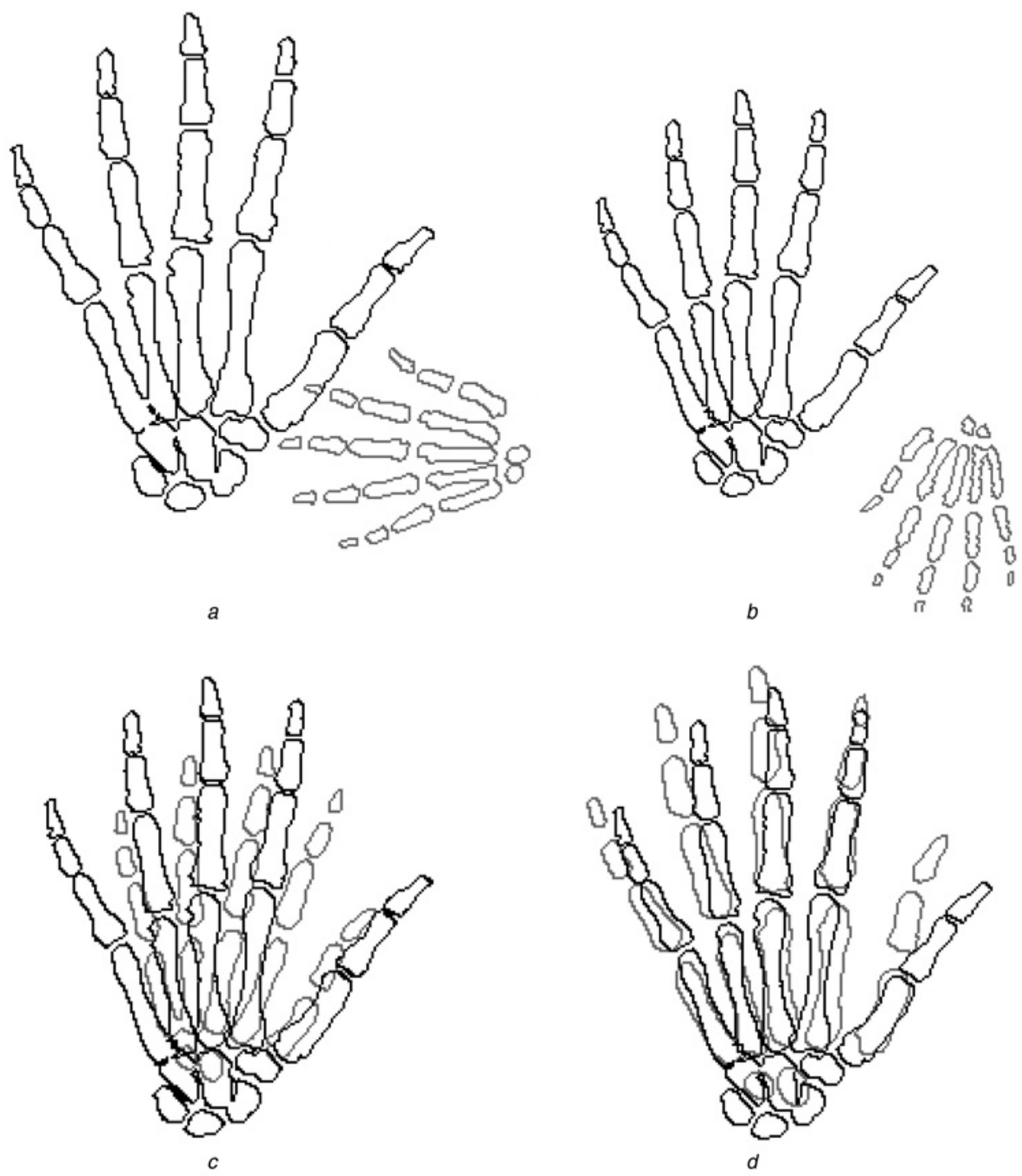

Fig. 3 Registration of two complex shapes containing various number of sub-shapes (bones)

$a$ Initial shapes before registration

$b$ Registration using the contour-based algorithm in [7]

$c$ Registration using the SDF-based algorithm in [8]

$d$ Registration using our registration algorithm

In Fig. 4, our registration method proposed here is compared with the SDF-based-registration algorithm investigated in [11]. Fig. $4 a$ shows two hand-bones before registration. Two shapes registered by the SDF-basedalgorithm in [11] are shown in Fig. $4 b$.

The two shapes registered by our registration method is depicted in Fig. 4c. A visual inspection of Fig. 4 indicates that the registration accuracy of the algorithm proposed here is more than that of the algorithm presented in [11] (e.g. compare the phalanges of the middle fingers of two registered hands in Figs. $4 b$ and $c$ ).

The distance term Dist for the two shapes in Fig. 4 before the registration is 9679. After the registration using the algorithm in [11], the distance term Dist decreases to 6616 . The registration technique presented in this paper on the other hand reduces the distance term Dist to 5462 implying a more accurate registration. The time taken by the algorithm of [11] is $2.42 \mathrm{~s}$, whereas the time elapsed by the CPU when using the registration algorithm proposed here is around $4.9 \mathrm{~s}$ during which the algorithm takes only two iterations to converge.

The aforementioned experiments demonstrate that the registration algorithm proposed here has the ability to register two shapes, even if there is no direct (one to one) correspondence among components forming the complex shapes.

To study the robustness of our algorithm at the presence of noise, binary noise is added to one of the clock shapes shown in Fig. $5 a$ to obtain the noisy shape of Fig. $5 b$ with $\mathrm{SNR}_{\mathrm{dB}}=$ -11.1 . The source shape before registration is also shown in Fig. 5c. As shown in Fig. 5d, the registration is achieved with good accuracy for complex shapes at the presence of the excessive noise.

The target shape shown in Fig. $5 a$ is contaminated with various amounts of binary noise to produce shape images with various SNRs. For a certain SNR, five different ensembles of binary noise are added to the target shape separately. For each noise ensemble, the registration 


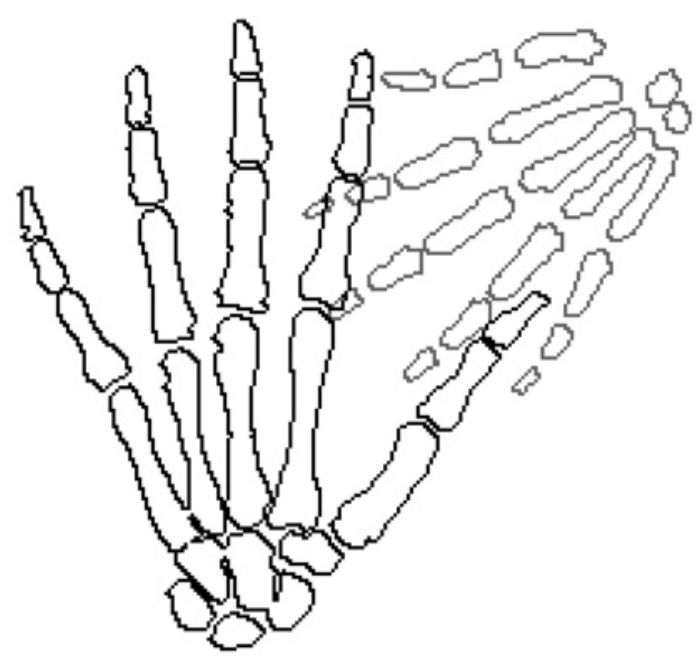

a
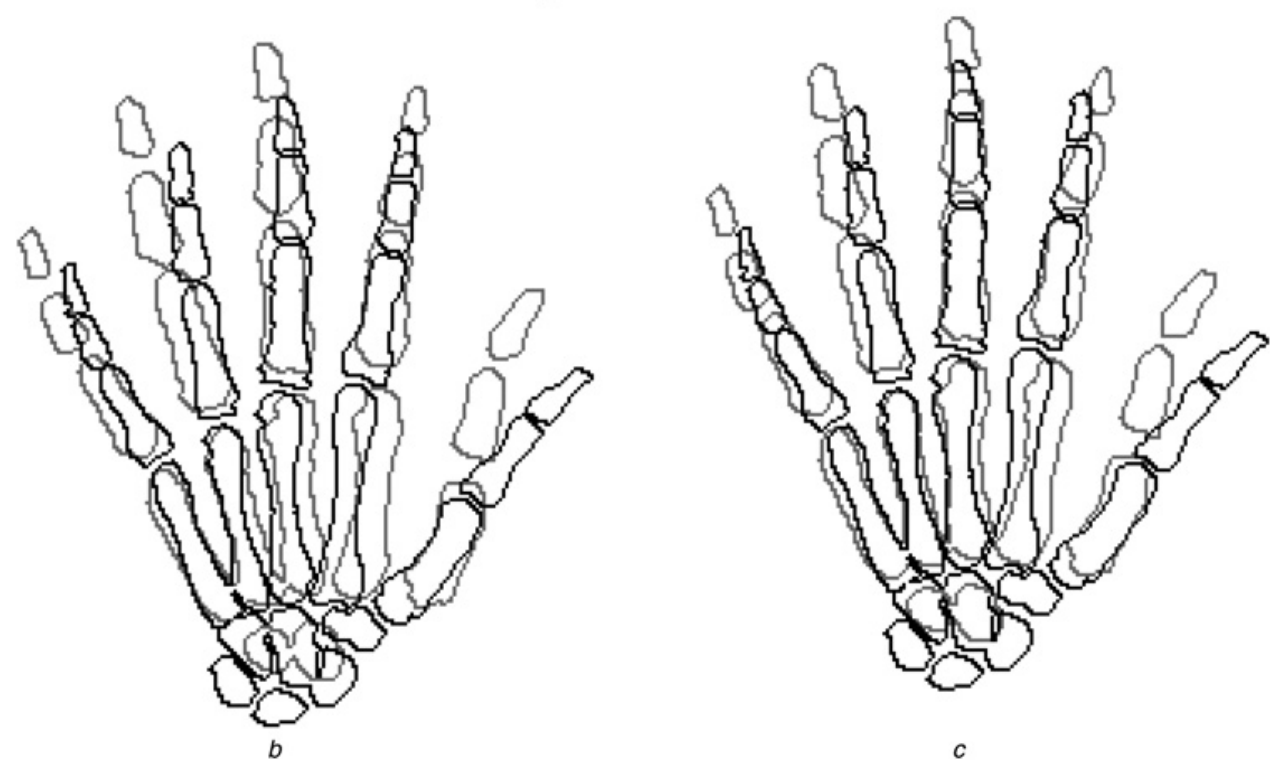

Fig. 4 Registration of two complex shapes containing various number of sub-shapes (bones)

$a$ Initial shapes before registration

$b$ Registration using SDF algorithm in [11]

$c$ Registration using the algorithm proposed here

between the noisy target shape and the source shape shown in Fig. $5 c$ is performed and then the mean and variance of the errors in rotation angle are measured for a certain SNR. The other registration parameters are assumed to be fixed with their optimal values in this experiment. The iterative projection algorithm [12] is also applied to the noisy target shapes to register with the source shape for comparison purposes. Fig. 6 depicts how the errors in rotation angle change by increasing the amount of binary noise (i.e. by lowering SNRs of the noisy target shape) for both algorithms. As shown in Fig. $6 a$, the registration algorithm proposed here outperforms the iterated projection in the presence of excessive noise in the target shape.

As depicted in Fig. $6 b$, our registration technique proposed in this paper requires significantly lower computational time than the iterated projection algorithm to register the noisy target shape and the source shape. The bottom of Fig. $6 b$ also shows the CPU elapsed time for registration by using our registration method in a more appropriate scale for a better visual inspection.

\subsection{Similar shapes with known transformations (accuracy of registration)}

In the next example, similar shapes transformed by using various known transformations are registered. This is to ensure that maximum accuracy is achievable and quantifiable. In Fig. 7, two different sets of transformation parameters are used to transform the source shape, which is a replica of the target shape. The algorithms in [7,8] and the algorithm proposed here are used to estimate the registration parameters. As shown in this figure, it is evident visually that the technique presented in this paper delivers the highest matching of the two identical shapes. These results are also presented in Table 1.

\subsection{Experiment on databases}

In this subsection, a set of $86 x$-ray images available from the database in the U.S. National Library of Medicine [17] are registered. These images are segmented to extract the shapes of the third cervical vertebra (C3). The algorithm 


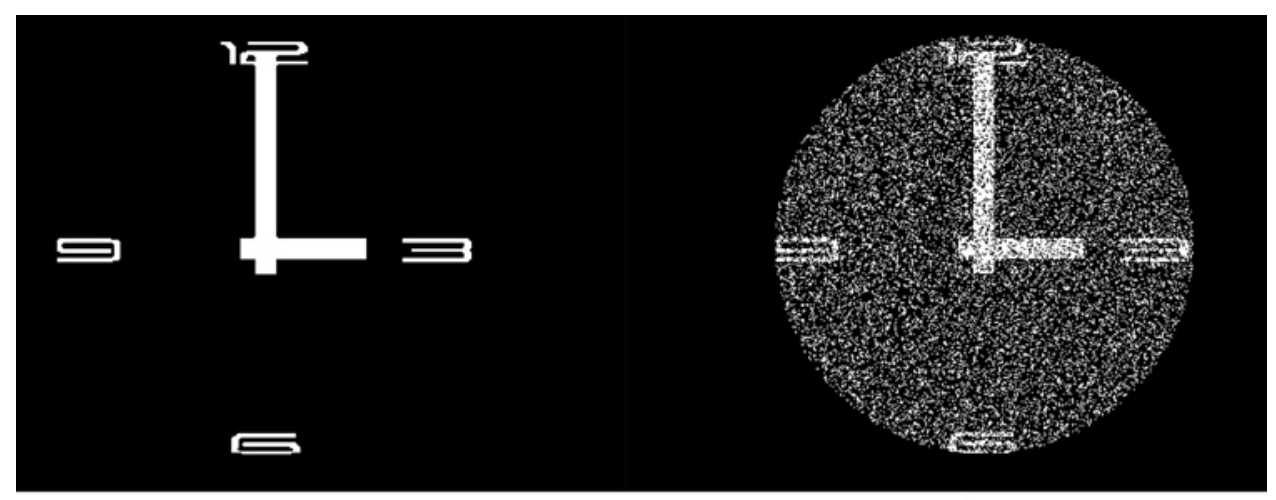

a

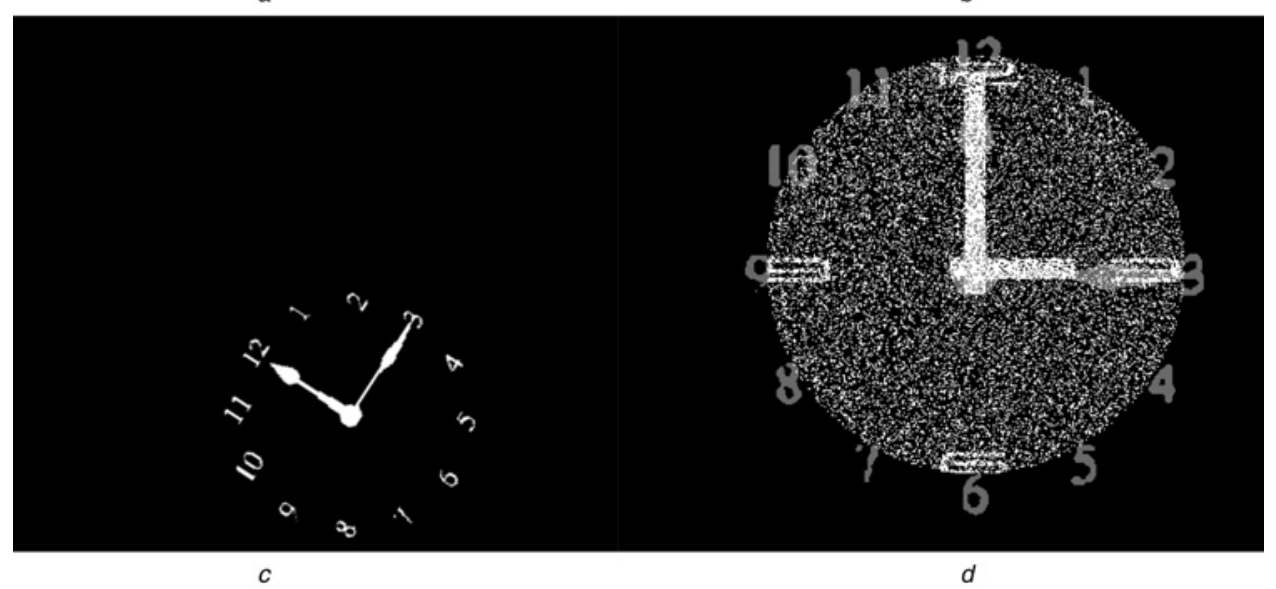

Fig. 5 Shape registration for complex shapes contaminated in binary noise

$a$ Original noiseless shape of a clock

$b$ Noisy shape of the clock shown in (a) $(\mathrm{SNR}=-11.1)$

$c$ Initial shape of another clock before registration

$d$ Shape registration result

proposed here along with the algorithms in [7, 8] are deployed to register these 86 shapes for the sake of performance comparison.

The quantitative assessment of this example is discussed in Fig. 8. This figure shows the average of the distance Dist between the target and the source shapes. For our method, this distance is reduced to $15.11 \%$ of the original distance before registration. It is also reduced to $15.38 \%$ for the algorithm in [7], and to $38.4 \%$ for the algorithm in [8].

This example demonstrates a better performance of our algorithm in registering vertebrae shapes in the database, while at the same time (as seen in Fig. 8b) our method outperforms the others in terms of the running time. The results summarised in Table 1 clearly suggest that in all of the experiments, the algorithm proposed here have produced better registration performance compared to the other two approaches. It is shown here that the proposed algorithm is capable of registering shapes with various Euler characteristic numbers leading to different topologies and higher shape complexity.

In the final part of this sub-section, the registration method proposed in this paper is applied on the shapes in the Swedish Leaf database. The number of sub-leaves in some shapes in this database varies from a shape to another. A contour-based registration method therefore fails to register these leaf shapes, since point correspondence between the contours of the two shapes cannot be established. The iterated projection method [12] is also applied to the Swedish Leaf database. The difference between shapes before and after the registration is calculated by employing the distance term Dist over the entire database. The results are presented in Table 2. As can be seen from the table, the average difference between shapes after the registration by using our registration method is lower than that for the iterated projection technique. The standard deviation of the shape differences after the registration for the method proposed here is significantly lower than that for the iterated projection algorithm. This lower standard deviation in shape differences for the registration method proposed here demonstrates its better reliability over a large database. As observed from this table, the registration technique proposed here is significantly faster than the iterated projection algorithm. The averaged elapsed CPU time over the entire database by using our method is $1.94 \mathrm{~s}$. On the other hand, the averaged CPU time taken by the iterated projection scheme over the whole database is $377.06 \mathrm{~s}$, which is significantly slower than our method proposed here.

\section{Conclusions}

This paper presents a shape registration algorithm which employs shape characteristic functions to represent shapes. Our algorithm succeeds to register complex shapes where the contour-based algorithm fails. Our registration algorithm is also significantly faster than the gradient descent-based algorithm employing SDFs [8] and the iterated projection-based method [12]. In all experiments performed 

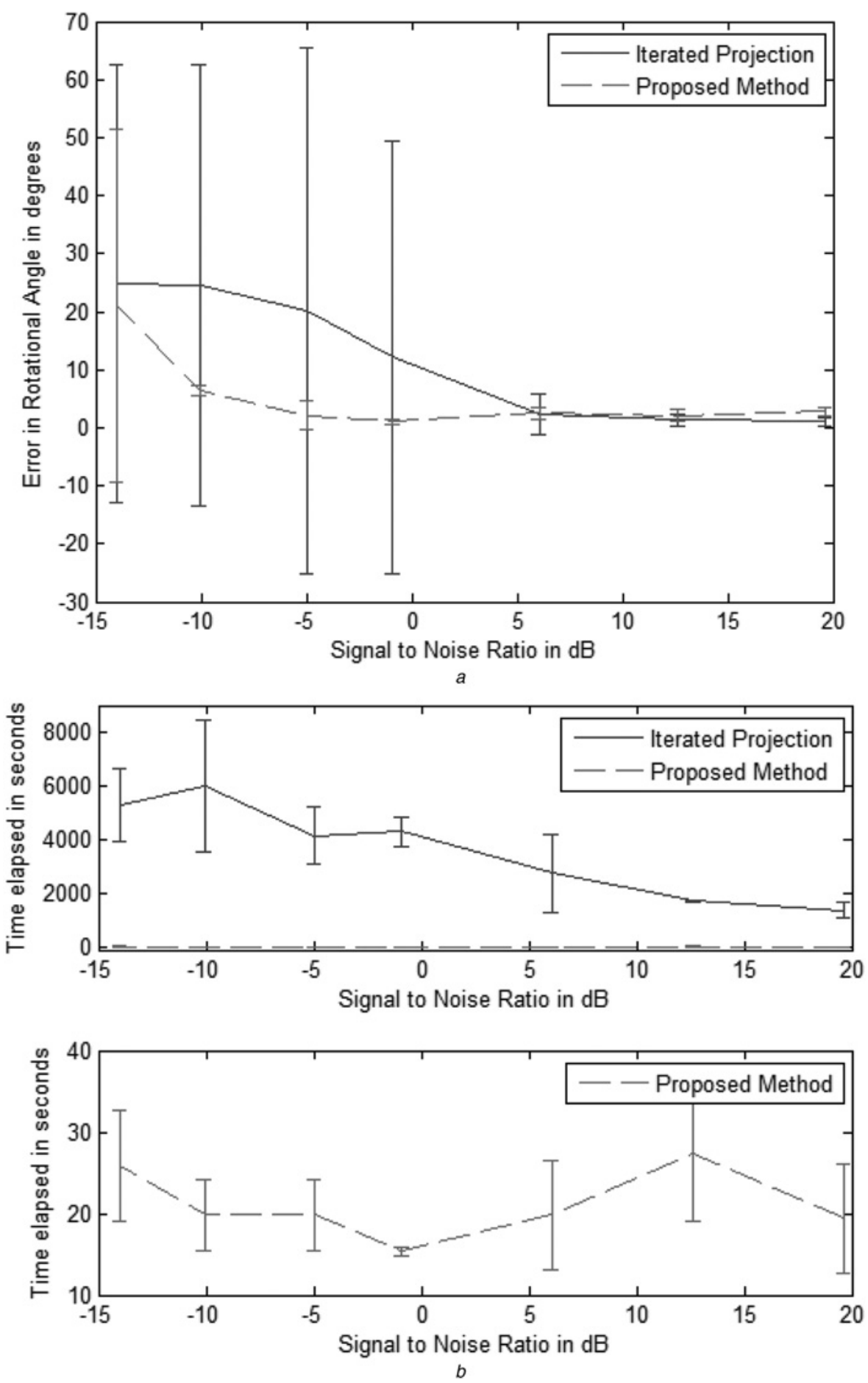

Fig. 6 Errors in rotational angle and CPU times elapsed for registration with respect to $S N R(d B)$ for the clock shapes shown in Fig. 4 while other parameters are considered to be fixed

$a$ Errors in rotational angle in degrees for the iterative projection algorithm (solid line) and the method proposed here (dashed line)

$b$ (top) CPU times elapsed for registration for the iterative projection algorithm (solid line) and the method proposed here (dashed line) (bottom) CPU times elapsed for registration for the method proposed here with an appropriate scale

throughout this paper, no local minima in our registration algorithm is observed. Our experiments presented in this paper also indicate that the shape registration algorithm proposed here is more robust than the iterated projection-based registration scheme at the presence of excessive noise. The results presented here suggest that the algorithm proposed here is much faster than the algorithm proposed in [8] and is as fast as the algorithm presented in [7]. The results presented in this section demonstrate a promising prospect for the shape registration algorithm proposed in this paper and indicate that this algorithm is robust and fast and can register shapes with various complexities.

Our algorithm takes advantage of Parseval theorem to estimate the rotation and the translation parameters using Fourier transform. The algorithm also uses the radial moments to estimate the scaling parameter. This registration technique is tested successfully on a variety of problems 


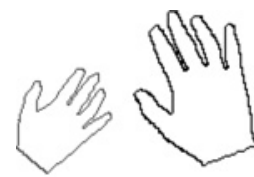

a

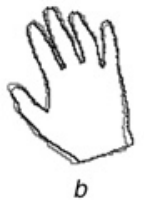

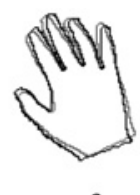

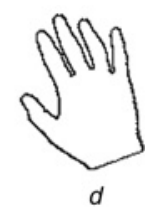

Fig. 7 Registration of identical shapes with synthetic transformations

$a$ Initial shapes, the source shape is generated by transforming the target shape using the parameters $\theta=-60, s=0.7, T_{x}=-90, T_{y}=20$

$b$ Registration of the shapes in (a) by using the approach in [7]

$c$ Registration of the shapes in (a) by using the method in [8]

$d$ Registration of the shapes in (a) by using our method
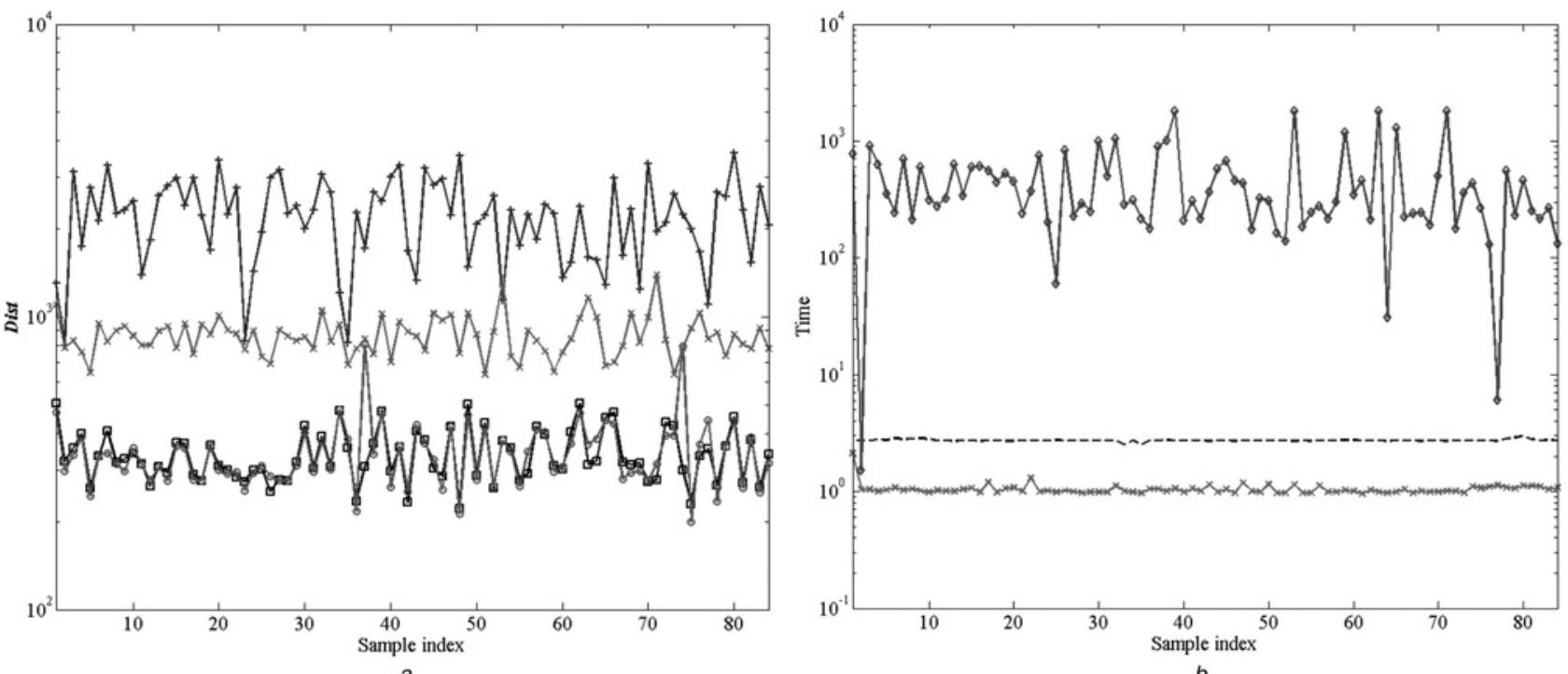

Fig. 8 Quantitative assessment of the registration results of shapes of the third cervical vertebra by using Dist and the time $t$

$a$ Comparison of Dist using original positions (plus-curve) with Dist produced by our algorithm (square-curve) and the algorithms in [7] (circle-curve) and [8] (star-curve)

$b$ Time required for registration using our algorithm (dashed-curve), and the algorithms in [7] (star-curve) and [8] (circle-curve)

Table 2 Average and standard deviation of the shape differences (Dist) as well as the average of the elapsed CPU time measured for the registration method proposed here and the iterated projection technique [12] applied on the Swedish Leaf database

\begin{tabular}{|c|c|c|c|}
\hline & $\begin{array}{c}\text { Average } \\
\text { of Dist }\end{array}$ & $\begin{array}{c}\text { Standard } \\
\text { deviation of } \\
\text { Dist }\end{array}$ & $\begin{array}{c}\text { Average } \\
\text { elapsed CPU } \\
\text { time (Sec) }\end{array}$ \\
\hline before registration & 3980.8 & 262.8 & - \\
\hline $\begin{array}{l}\text { after registration } \\
\text { with the method } \\
\text { proposed in this } \\
\text { paper }\end{array}$ & 1972.4 & 362.6 & 1.94 \\
\hline $\begin{array}{l}\text { after registration } \\
\text { with the iterated } \\
\text { projection technique }\end{array}$ & 2071.6 & 940.5 & 377.06 \\
\hline
\end{tabular}

including complex shapes and shapes with various topologies, which cannot be registered using contour-based methods. The experimental results demonstrate that the proposed registration algorithm is fast, accurate, stable, and according to our experiments, does not fall into local minima in contrast with other registration algorithms in the literature. Furthermore, our technique is successfully exploited to register shapes in the databases of the Swedish Leaves and the U.S. National Library of Medicine with a better performance in comparison with the contour-based and iterative projection-based registration algorithms. The algorithm proposed here therefore can be a solution to a wide range of shape registration problems encountered in image processing and computer vision especially medical problems. As a future work, this algorithm can be generalised to register $2 \mathrm{D}$ and $3 \mathrm{D}$ volumetric grey-scale images.

\section{References}

1 Mahmoodi, S.: 'Shape-based active contours for fast video segmentation', IEEE Signal Process. Lett., 2009, 16, (10), pp. 857-860

2 Chan, T., Zhu, W.: 'Level set based shape prior segmentation'. Technical Report 03-066, Computational Applied Mathematics, UCLA, Los Angles, 2003

3 Riklin-Raviv, T., Kiryati, N., Sochen, N.: 'Unlevel sets: geometry and prior-based segmentation'. European Conf. Computer Vision (ECCV), 2004, vol. 3024, pp. 50-61

4 Charpiat, G., Faugeras, O., Keriven, R.: 'Approximations of shape metrics and application to shape warping and empirical shape statistical', J. Found. Comput. Math., 2005, 5, (1), pp. 1-58

5 Cremers, D., Osher, S., Soatto, S.: 'Kernel density estimation and intrinsic alignment for shape priors in level set segmentation', Int. J. Comput. Vis., 2006, 69, pp. 335-351 


\section{www.ietdl.org}

6 Marques, J.S., Abrantes, A.J.: 'Shape alignment - optimal initial point and pose estimation', Pattern Recognit. Lett., 1997, 18, pp. 49-53

7 Markovsky, I., Mahmoodi, S.: 'Least-squares contour alignment', Signal Process. Lett., 2009, 16, pp. 41-44

8 Paragios, N., Rousson, M., Ramesh, V.: 'Non-rigid registration using distance functions', Comput. Vis. Image Understand., 2003, 89, pp. 142-165

9 Paragios, N., Rousson, M., Ramesh, V.: 'Matching distance functions: a shape-to-area variational approach for global-to-local registration'. Presented at the Proc. Seventh European Conf. Computer Vision-Part II, 2002

10 Al-Huseiny, M.S., Mahmoodi, S., Nixon, M.S.: 'Robust rigid shape registration method using a level set formulation'. Lecture Notes in Computer Science - Proc. Sixth Int. Symp. Visual Computing, 2010, pp. 252-261

11 Mahmoodi, S., Al-Huseiny, M., Nixon, M.S.: 'Similarity registration for shapes based on signed distance functions'. Lecture Notes in Computer
Science - Proc. Eighth Int. Symp. Visual Computing, 2012, pp. 599-609

12 Cremers, D., Schmidt, F.R., Barthel, F.: 'Shape priors in variational image segmentation: convexity, Lipschitz continuity and global optimal solutions'. IEEE Int. Conf. Computer Vision and Pattern Recognition, 2008, pp. 1-6

13 Liu, W., Mahmoodi, S., Havelock, T., Bennett, M.: 'Robust similarity registration technique for volumetric shapes represented by characteristic functions', Pattern Recognit., 2014, 47, (3), pp. 1144-1158

14 Casasent, D., Psaltis, D.: 'Position, rotation, and scale invariant optical correlation', Appl. Opt., 1976, 15, pp. 1795-1799

15 Viola, P., Wells, W.M.: 'Alignment by maximization of mutual information', Int. J. Comput. Vis., 1997, 24, pp. 137-154

16 Mukundan, R.: 'A comparative analysis of Radial-Tchebichef moments and Zernike moments'. British Machine Vision Conference, 2009

17 U. S. National Library of Medicine. NHANES II [online]. Available: http://archive.nlm.nih.gov/proj/ftp/ftp.php\#available 